This document is the accepted manuscript version of the following article: Muoth, C., Wichser, A., Monopoli, M., Correia, M., Ehrlich, N., Loeschner, K., ... Buerki-Thurnherr, T. (2016). A 3D co-culture microtissue model of the human placenta for nanotoxicity assessment. Nanoscale, 8(39), 17322-17332. https://doi .org/10.1039/c6NR06749B

\title{
A 3D microtissue co-culture model of the human placenta for nanotoxicity assessment
}

Carina Muoth ${ }^{a}$, Adrian Wichser ${ }^{a}$, Marco Monopoli ${ }^{b, \#}$, Manuel Correia ${ }^{c}$, Nicky Ehrlich ${ }^{c, \S}$, Katrin Loeschnerc, Audrey Galludd, Melanie Kuckia, Liliane Dienera, Pius Manser ${ }^{\mathrm{a}}$, Wolfram Jochum ${ }^{\mathrm{e}}$, Peter Wick ${ }^{\mathrm{a}}$ and Tina Buerki-Thurnherr ${ }^{a,}{ }^{*}$

There is increasing evidence that certain nanoparticles (NPs) can overcome the placental barrier, raising concerns on potential adverse effects on the growing fetus. But even in the absence of placental transfer, NPs may pose a risk to proper fetal development if they interfere with the viability and functionality of the placental tissue. The effects of NPs on the human placenta are not well studied or understood, and predictive in vitro placenta models to achieve mechanistic insights on NP-placenta interactions are essentially lacking. Using the scaffold-free hanging drop technology, we developed a wellorganized and highly reproducible 3D co-culture microtissue (MT) model consisting of a core of placental fibroblasts surrounded by a trophoblast cell layer, which resembles the structure of the in vivo placental tissue. We could show that secretion levels of human chorionic gonadotropin (hCG) were significantly higher in 3D than in 2D cell cultures, which indicates an enhanced differentiation of trophoblasts grown on 3D MTs. NP toxicity assessment revealed that cadmium telluride (CdTe) and copper oxide $(\mathrm{CuO})$ NPs but not titanium dioxide $\left(\mathrm{TiO}_{2}\right)$ NPs decreased MT viability and reduced the release of hCG. NP acute toxicity was significantly reduced in 3D co-culture MTs compared to 2D monocultures. Taken together, 3D placental MTs provide a new and promising model for the fast generation of tissue-relevant acute NP toxicity data, which are indispensable for the safe development of NPs for industrial, commercial and medical applications

\section{Introduction}

The developing fetus is highly vulnerable to toxic substances and adverse effects occur at doses that are usually not of any concern for adult individuals. To protect the growing fetus from potential harm, a key function of the placenta is to act as a tight barrier for the passage of pathogens and toxic compounds. However, there is increasing evidence that nanoparticles (NPs) can overcome this protective barrier and exert fetotoxic effects. Recent animal studies described placental uptake and/or translocation of gold $(\mathrm{Au})$, silica $\left(\mathrm{SiO}_{2}\right)$, polystyrene $(\mathrm{PS})$, cadmium oxide (CdO), titanium dioxide $\left(\mathrm{TiO}_{2}\right) \mathrm{NPs}$, quantum dots (Qdots) or carbon nanotubes (CNTs) among others (reviewed by ${ }^{1,2}$ ). Direct or indirect fetotoxic effects were observed after the exposure to multi-walled CNTs (MWCNTs) ${ }^{3,4}$, single-walled CNTs (SWCNTs) 5, 6, CdO 7, cadmium telluride $(\mathrm{CdTe})^{8}$ or $\mathrm{Cu} \mathrm{NPs}{ }^{9}$. On the other hand, NPs hold great promises for the development of targeted therapies to treat patients more effectively and with reduced or absent off-target effects. In pregnancy, nanomedicine offers novel therapeutic approaches to specifically treat pregnant 
women, placental complications or fetal diseases by either preventing or enabling placental uptake or translocation of drug-loaded NPs ${ }^{2}{ }^{10}$.

To ensure the safe development of NPs for various applications, a profound knowledge on NP effects and uptake mechanisms at the human placental barrier is crucial. Since the placenta is the most species-specific mammalian organ with unique anatomical and physiological properties 11 , it is difficult to extrapolate from animal data to humans. A well-established model with a high predictability of in vivo placental transfer is the ex vivo perfusion of human full term placentas ${ }^{12}$ 13. However, this model has certain drawbacks such as the restricted perfusion time of approximately 6 - $8 \mathrm{~h}$, the low rate of successful perfusions as well as limited possibilities for mechanistic studies. In contrast, 2D in vitro cell culture models allow high-throughput studies but do not properly mimic the in vivo situation as they lack the complex organization and environment of the placental tissue such as the close interaction of different cell types or maternal and fetal blood flow. Hence, it is not surprising that 2D cultures often do not accurately predict in vivo toxicity and biological effects of NPs ${ }^{14}$. Moreover, key aspects of tissue distribution and penetration of NPs cannot be adequately addressed if cells are cultivated on artificial flat and rigid 2D surfaces.

Therefore, there is an urgent need for a predictive organotypic in vitro model of the human placenta that enables rapid nanotoxicity assessment of a large variety of different NPs as well as mechanistic studies on tissue uptake and penetration. A promising alternative to conventional 2D monocultures are co-culture microtissues (MTs). They provide extensive 3-dimensional cellcell and cell-matrix interactions that promote the recovery of original tissue morphology and functionality ${ }^{15-17}$. Biological functions that were shown to be more similar to the in vivo situation in 3D MTs include cell proliferation, differentiation, morphogenesis, gene expression or drug response ${ }^{18-21}$. In the field of nanobiotechnology, MTs are increasingly used for toxicity and tissue penetration studies of different nanomaterials ${ }^{22}$. For instance, multiple exposure of liver MTs to silver ( $\mathrm{Ag}$ ) or zinc oxide ( $\mathrm{nnO}$ ) NPs was associated with more significant adverse effects as compared to amine-modified $\mathrm{TiO}_{2}$ NPs or MWCNTs ${ }^{23}$. Luo et al. investigated cytotoxic effects of bismuth (Bi) NPs with different surface modifications on 2D monolayer cultures and 3D MTs either consisting of epithelial cells (HeLa cells) or bone fibroblasts (MG-63 cells). Toxic effects on 3D MTs were significantly lower than on 2D monolayers ${ }^{24}$. This increased resistance to toxic substances in 3D MTs was confirmed by Lee et al. after the exposure of liver MTs to Qdots and Au NPs ${ }^{14}$. MT penetration studies showed a size-dependent penetration of carboxylate PS beads into tumor MTs, which could be significantly increased by collagenase treatment of MTs or immobilization of collagenase to the NP surface. Significant uptake was observed for 20 and $40 \mathrm{~nm}$ PS beads while less transport was detected for 100 and $200 \mathrm{~nm}$ PS beads ${ }^{25}$. Similarly, size-dependent penetration into breast cancer MTs (MCF-7 cells) was detected for 2 and $6 \mathrm{~nm}$ tiopronin- 
coated Au NPs whereas $15 \mathrm{~nm}$ NPs were localized in the outer rim of the MTs ${ }^{26}$. For 15 and 55 $\mathrm{nm}$ block copolymer micelles, Mikhail et al. found a size- and time-dependent penetration into cervical (HeLa) and colon (HT29) cancer MTs after 1 and $24 \mathrm{~h}$ but with much higher fluorescence signal intensity in HeLa MTs ${ }^{27}$. In summary, 3D MT models offer a promising approach to close the gap between 2D cell cultures and animal studies and to support the identification of safe nanomaterials for various applications.

In this study, we developed and characterized a high-throughput compatible in vitro co-culture MT model of the human placental barrier using the hanging drop technology ${ }^{28}$. MTs consisting of a core of human villous mesenchymal fibroblasts (HVMF) surrounded by a layer of human trophoblastic choriocarcinoma cells (BeWo cells) were generated and characterized with regard to MT morphology, growth, viability and expression of cell type-specific markers. Levels of human chorionic gonadotropin ( $\mathrm{hCG}$ ), an essential pregnancy-related hormone exclusively produced by trophoblasts, were compared between 3D MTs and BeWo cells grown on 2D plastic cell culture substrates. To evaluate the use of this model for nanosafety assessment, MT viability and functionality were investigated after the exposure to $\mathrm{TiO}_{2}, \mathrm{CdTe}-\mathrm{COOH}$ or $\mathrm{CuO} \mathrm{NPs}$ for $24 \mathrm{~h}$ and viability data were compared to 2D monolayer cultures.

\section{Experimental section \\ Cell culture}

BeWo cells (b30 clone), a human placental choriocarcinoma cell line with cytotrophoblastic characteristics, were obtained from Prof. Dr. Ursula Graf-Hausner (Zurich University of Applied Sciences, Wädenswil, Switzerland) with permission of Dr. Alan L. Schwartz (Washington University School of Medicine, MO, USA) and cultured in Ham's F-12K medium (Gibco, Luzern, Switzerland) supplemented with $10 \%$ fetal calf serum (FCS, Invitrogen, Basel, Switzerland), 2 mM L-Glutamine (Gibco, Luzern, Switzerland) and $1 \%$ penicillin/streptomycin (Gibco, Luzern, Switzerland). BeWo cells between passages 18 to 38 were used for experiments. Primary human villous mesenchymal fibroblasts (HVMF, PromoCell) were cultured in fibroblast growth medium 2 (FGM, PromoCell) supplemented with $2 \mathrm{mM} \mathrm{L-Glutamine} \mathrm{(Gibco,} \mathrm{Luzern,} \mathrm{Switzerland),} 1 \%$ penicillin/streptomycin (Gibco, Luzern, Switzerland) and one vial of SupplementMix (PromoCell). Primary HVMFs were used for experiments between passages 4 and 10. HTR-8/SVneo cells originating from the extravillous cytotrophoblast were obtained from Dr. Charles H. Graham (Queen University, Canada) 29. Cells were maintained in RPMI (Gibco, Luzern, Switzerland) supplemented with 5 \% FCS (Invitrogen, Basel, Switzerland), 2 mM L-Glutamine (Gibco, Luzern, Switzerland) and $1 \%$ penicillin/streptomycin (Gibco, Luzern, Switzerland)). All cell lines were cultivated in a humidified incubator at $37{ }^{\circ} \mathrm{C}$ with $5 \% \mathrm{CO}_{2}$ atmosphere and were sub-cultured twice a week. 


\section{Co-culture microtissue formation}

The scaffold-free hanging drop system purchased from Insphero AG (Schlieren, Switzerland) was used to generate 3D co-culture MTs. HVMF were seeded into the GravityPLUS ${ }^{\text {TM }} 96$ well plates (CInSphero AG, Switzerland) at a density of 5000 cells $/ 40 \mu$ ldrop volume). MT formation was performed in FGM containing 20 \% FCS. At day 3, 300 BeWo or 500 HTR-8/SVneo cells in a volume of $20 \mu \mathrm{l}$ were added to the fibroblastic core MTs. At day 5, MTs were transferred into GravityTRAPTM 96 well plates (CInSphero AG Switzerland) for subsequent NP exposure or analysis. Medium was replaced every second to third day. The mean geometric diameter of spheroids was calculated according to the equation: $(a \times b)^{1 / 2}$, where $a$ and $b$ are the measured orthogonal diameters ${ }^{30}$.

\section{Nanoparticle synthesis and dispersion}

$\mathrm{TiO}_{2}$, CdTe surface-functionalized with carboxylate moieties $(\mathrm{CdTe}-\mathrm{COOH})$ and $\mathrm{CuO}$ NPs were provided within the EU FP7 Nanosolutions project (http://www.nanosolutions.eu). Briefly, $\mathrm{TiO}_{2}$ NPs were synthesized by forced hydrolysis of titanium tetrachloride solution followed by a washing step, stabilization with nitric acid and drying under vacuum at mild temperatures. CdTe$\mathrm{COOH}$ NPs were manufactured by reacting $\mathrm{Cd}$ and Te precursors in aqueous solution in presence of mercaptocarboxylic acid as a stabilizing ligand. Upon reaching the desirable emission wavelength, quantum dots were precipitated by isopropanol. Several washing-centrifugations were followed by drying at $50{ }^{\circ} \mathrm{C}$ under vacuum to yield a dry powder. CuO NPs were fabricated in a two-step procedure including the synthesis of the freshly precipitated calcium hydroxycarbonate and its drying and calcination at the controlled heating rate.

Stock suspensions of $5 \mathrm{mg} / \mathrm{ml}$ of $\mathrm{TiO}_{2}$ or $\mathrm{CdTe}-\mathrm{COOH}$ NPs in ultra-pure water were obtained by bath sonication (1 min, 600 W, Sonorex RK156 BH, Bandelin). CuO NPs were dispersed in ultrapure water to a stock suspension of $1 \mathrm{mg} / \mathrm{ml}$ using a probe sonicator operating at $230 \mathrm{~V} / 50 \mathrm{~Hz}$ (Branson Sonifier 250, Branson Ultrasonic Co., Danbury, CT, USA, probe diameter of $6.5 \mathrm{~mm}$, maximum peak-to-peak amplitude of $247 \mu \mathrm{m}$ ). The CuO NPs suspension was treated for $5 \mathrm{~min}$ at $30 \%$ of the maximum peak-to-peak amplitude, corresponding to a specific acoustic energy of $0.39 \mathrm{~kJ} / \mathrm{dm}^{3}$ as determined by a calorimetric method for probe sonicator calibration ${ }^{31}$. Due to the potential dissolution of $\mathrm{CdTe}-\mathrm{COOH}$ and $\mathrm{CuO} N \mathrm{NP}$, stock suspensions were prepared just prior to the experiments, diluted to the required final concentrations in supplemented FGM and immediately added to the placental co-culture MTs. NP powder and suspensions were characterized using different analytical methods (see Electronic supplementary information (ESI) for a description of the NP characterization methods and Table S1 for a summary of the NP characteristics). 


\section{ATP assay}

To determine the effects of different NPs on MT viability, MTs were exposed to 3, 6, 12, 25 and $50 \mu \mathrm{g} / \mathrm{ml} \mathrm{TiO}_{2}, \mathrm{CdTe}-\mathrm{COOH}$ or $\mathrm{CuO}$ NPs for $24 \mathrm{~h}$ (final volume $70 \mu \mathrm{l}$ ). $0.1 \%$ Triton X-100 was used as the positive control and FGM as the medium control. Intracellular ATP levels were determined using a CellTiter-Glo ${ }^{\circledR}$ Luminescent Cell Viability Assay (Promega, USA), which is specifically adapted to 3D MTs. Samples were washed with PBS before addition of $40 \mu \mathrm{l} /$ well of a 50:50 FGM/CellTiter-GLO ${ }^{\circledR}$ mixture and incubation for $30 \mathrm{~min}$ on a shaker at room temperature (RT). Cell lysates were transferred to a black 96 -well plate (Microfluor ${ }^{\mathrm{TM}} 1$, Thermoscientific, New York, USA) together with an ATP standard (Sigma, Buchs, Switzerland). Luminescent signals were measured with a microplate reader (Mithras ${ }^{2}$ LB 943 Monochromator Multimode Reader, Berthold Technologies, Zug, Switzerland). Values were expressed in percentage of untreated control samples.

For viability assays with 2D BeWo and HVMF cultures, cells were seeded in 96-well plates at a density of $10^{\prime} 000$ or 8000 cells/well in $200 \mu \mathrm{FGM}$, respectively. After overnight incubation, cells were exposed to $3-50 \mu \mathrm{g} / \mathrm{ml} \mathrm{TiO}_{2}$, CdTe-COOH or CuO NPs for $24 \mathrm{~h}$. The ATP assay was performed as described for 3D co-culture MTs but with the addition of $100 \mu$ l of the 50:50 FGM/CellTiter-GLO ${ }^{\circledR}$ mixture.

\section{Immunohistochemical characterization of 3D MTs}

For immunohistochemical (IHC) staining, 30 - 40 MTs were pooled, fixed with $4 \%$ formalin, embedded in paraffin and cut into $1.5-2 \mu \mathrm{m}$ thick tissue sections. The IHC staining was performed using an automated staining system (BOND-MAX, Leica Microsystems). Hematoxylin and eosin (HE) staining was performed according to the standard protocol. For the antibody staining, sections were incubated with either MFN116 (1:400, 15 min, DAKO M0821), vimentin (1:1000, 15 min, DAKO M0725) or MIB-1 (1:60, 30 min, DAKO M7240) followed by visualization with 3,3'Diaminobenzidine and counterstaining with hematoxylin (Bond Polymer Refine Detection Kit, Leica Microsystems). Prior to the antibody staining, antigen retrieval was performed using the Leica Bond Enzyme Pretreatment Kit for MNF116 or Leica Bond Epitope Retrieval Solution 2 for vimentin and MIB-1. The stained slices were captured with a Leica DM4000 B LED microscope (Leica Microsystems).

\section{Live/Dead staining}

Cell viability of MTs was assessed using a Live/Dead Cell Imaging kit (R37601, Molecular Probes). MTs were exposed to $0.5,1,3$ and $12 \mu \mathrm{g} / \mathrm{ml}$ CuO NPs for $24 \mathrm{~h}$ and immediately stained according 
to the manufacturer's protocol. Z-stack images were subsequently acquired with a 10x magnification objective on a confocal laser scanning microscope (LSM 780, Zeiss, Feldbach, Switzerland). Viable cells appeared green whereas dead cells appeared red.

\section{hCG ELISA}

The production of hCG was measured in 2D BeWo cell cultures and 3D co-culture MTs in the absence or presence of $20 \mu \mathrm{M}$ forskolin (Sigma, Buchs, Switzerland). After transferring the MTs to trap plates, $70 \mu \mathrm{l}$ of fresh FGM or FGM supplemented with $20 \mu \mathrm{M}$ forskolin was added. Supernatants of four MTs per concentration were pooled and stored at $-80^{\circ} \mathrm{C}$. For $2 \mathrm{D}$ BeWo cultures, cells were seeded in 96-well plates at a density of 3000 cells/well in $70 \mu \mathrm{l} \mathrm{FGM} \mathrm{and} \mathrm{incubated} \mathrm{for}$ $48 \mathrm{~h}$. Medium was thereafter replaced with fresh FGM or FGM supplemented with $20 \mu \mathrm{M}$ forskolin. After $24 \mathrm{~h}$, supernatants of four wells with the same treatment were pooled and stored at $-80^{\circ} \mathrm{C}$. The effect of NPs on hCG production in 3D co-culture MTs was determined after treatment with $3-25 \mu \mathrm{g} / \mathrm{ml} \mathrm{CdTe}-\mathrm{COOH}, \mathrm{CuO}$ or $\mathrm{TiO}_{2} \mathrm{NPs}$ for $24 \mathrm{~h}$ in FGM supplemented with 20 $\mu \mathrm{M}$ forskolin. To measure hCG production, the protocol from Malek et al. was adapted to MTs 32. High protein binding 96 well plates (Corning, Root, Switzerland) were coated with polyclonal rabbit anti-human CG (Dako, A0231) in $50 \mathrm{mM} \mathrm{NaHCO}_{3}(1: 1000)$ over night at $4{ }^{\circ} \mathrm{C}$. Blocking was performed with $0.5 \%$ bovine serum albumin (BSA) in PBS during $2 \mathrm{~h}$ at RT and plates were washed three times with $0.1 \%$ Tween 20 in PBS before standard or supernatants were added to the wells for $90 \mathrm{~min}$ at $37^{\circ} \mathrm{C}$. After three washing steps, the plates were incubated with mouse anti-human CG (BioSupply, UK) diluted in $1 \%$ BSA in PBS (1:5000) for $90 \mathrm{~min}$ at $37^{\circ} \mathrm{C}$ followed by three washing steps. Goat anti-mouse-IgG-horse radish peroxidase conjugate (BioRad, Cressier, Switzerland) diluted in $1 \%$ BSA in PBS (1:5000) was added to the wells for 90 min at $37^{\circ} \mathrm{C}$ followed by four washing steps. A 0-phenylenediamine dihydrochloride I (OPD) tablet of $20 \mathrm{mg}$ (Sigma, Buchs, Switzerland) was dissolved in $21 \mathrm{ml} 0.05 \mathrm{M}$ phosphate-citrate buffer with $0.03 \%$ sodium perborate ( $\mathrm{pH}$ 5.0, Sigma, Buchs, Switzerland) and $100 \mu \mathrm{l}$ of the solution were added to each well for $18 \mathrm{~min}$ at RT before the reaction was stopped with $2 \mathrm{~N} \mathrm{H}_{2} \mathrm{SO}_{4}$. Absorbance was measured at $492 \mathrm{~nm}$ with a microplate reader (Mithras² LB 943 Monochromator Multimode Reader, Berthold Technologies, Zug, Switzerland). Concentration linearity for the standard was given between 0 and $30 \mathrm{mU} / \mathrm{ml}$. Values were blank-corrected.

\section{Scanning Electron Microscopy (SEM)}

SEM was used to evaluate the surface morphology of the co-culture MTs. Briefly, MTs were fixed with modified Karnovsky solution (containing $4 \%$ paraformaldehyde, $2.5 \%$ glutaraldehyde and 50:50 mix of $d_{d H_{2}} \mathrm{O}$ and PBS) at RT for $1 \mathrm{~h}$. Samples were washed twice with PBS, followed by a dehydration series in ethanol (50 - $100 \%)$. MTs were placed on glass cover slips and exposed to 
hexamethyldisilazane (HMDS) for 30 min before drying at RT. Samples were stored in a vacuumassisted desiccator until sputter-coating with a $10 \mathrm{~nm}$ thick layer of gold-palladium (Au/Pd: 80/20) alloy. Analysis was performed with a scanning electron microscope (Hitachi S-4800, Japan).

\section{Transmission Electron Microscopy (TEM)}

MTs were fixed in $3 \%$ glutaraldehyde in $0.1 \mathrm{M}$ sodium cacodylate buffer and washed in $0.2 \mathrm{M}$ sodium cacodylate buffer. After a post-fixation step in $2 \%$ osmium tetroxide in $0.1 \mathrm{M}$ sodium cacodylate buffer, samples were dehydrated through a graded ethanol series followed by acetone and finally embedded in Epon resin (Sigma, Buchs Switzerland). Ultrathin sections were contrasted with 2 \% uranyl acetate and lead citrate (Reynolds 1963) before imaged in a Zeiss EM 900 (Carl Zeiss Microscopy, GmbH, Germany) at 80 kV.

\section{Statistical analysis}

Data are represented as the mean \pm standard error of the mean (SEM) from at least three independent experiments. Statistical significance was determined using a two-tailed Student's t-test. A p-value below 0.05 was considered to be statistically significant. The web application WebAPP MDRA (available at http://biostatistics.dkfz.de/mdra/, access date: $30^{\text {th }}$ of May 2016) was used to calculate $\mathrm{EC}_{50}$ values.

\section{Results and discussion}

\section{Establishment and characterization of a human placental co-culture MT model}

To develop a reproducible 3D in vitro co-culture MT model of the human placenta we employed the scaffold-free hanging drop technology (GravityPLUS ${ }^{\mathrm{TM}}$ plates). Compared to other methods used to generate 3D MTs (e.g. natural or synthetic hydrogels, solid scaffolds, rotary cultures or liquid overlay) the hanging drop technology allows for the precise and reproducible control of MT size and composition via the cell seeding number. Moreover, co-culturing is feasible and direct contact between different cell types enables their natural intercellular organization, polarization and development of an extracellular matrix. The 96-well format makes this technique compatible with high-throughput assay systems and thus offers an interesting method to screen various drugs or NPs on different tissue models 33,34 . In the human term placenta, fetal capillaries are embedded in villous trees that are in direct contact with maternal blood (Figure 1). In order to enter the fetal blood stream, NPs have to cross the trophoblast cell layer that confines the villous trees, the villous stroma containing fibroblasts as well as the fetal capillary endothelium 35, 36. Trophoblasts are among the most important cells of the placental barrier and express highly 
specialized importers and exporters to regulate the transfer of gases, nutrients, waste products and different toxic substances. Moreover, they secrete a variety of hormones that are essential to fetal life and placental development. To mimic the human placental tissue in a 3D co-culture MT model, we established fibroblastic core MTs from HVMFs that were surrounded by an outer layer of trophoblasts (Figure 1). For the latter, the well-characterized and widely used choriocarcinoma cell line BeWo was chosen since these cells reveal many of the morphological and functional characteristics of villouos trophoblasts, including partial syncytial fusion, syncytin 1 and 2 regulation, expression of transporters as well as secretion of hormones such as hCG, human placental lactogen, progesterone and estradiol ${ }^{37-39}$. Furthermore, BeWo cells are of human origin, which is important to circumvent species-specific differences in in vitro placenta models. The optimal medium that best sustained the viability of HVMFs and BeWo cells was FGM (data not shown). To obtain well-structured co-culture MTs, we first established the formation of a stable fibroblastic core MT. Cell seeding number and incubation time for HVMFs were optimized in order to obtain tightly packed core MTs that resisted major invasion by BeWo cells. Spheroid sizes should not exceed $500 \mu \mathrm{m}$ in order to avoid necrotic cores due to diffusion limitations for oxygen and nutrients ${ }^{20}$. Stable core MTs with a highly reproducible size of $223 \pm 8.3 \mu \mathrm{m}$ were obtained 3 days after seeding of 5000 HVMFs per well of a 96 -well hanging drop plate. To obtain a continuous BeWo cell coat around the core MTs, 300 BeWo cells were added to the fibroblastic core MTs and further cultivated in the hanging drop for 2 days. After 5 days, MTs were transferred into non-adhesive GravityTRAPTM plates, fixed and subjected to morphological analysis. Highly spherical and compact spheroids were formed as evidenced by SEM analysis of the MT surface (Figure 2A). TEM micrographs further revealed that BeWo cells exhibited a polarized morphology with numerous microvilli on their apical cell surface (Figure 2B). Moreover, they developed tight junctional complexes and desmosomes between neighboring cells (Figure 2B). Immunohistochemical staining of paraffin sections for vimentin (marker of fibroblasts) and cytokeratin (marker of trophoblasts) confirmed the formation of highly organized co-culture MTs with a distinct coreshell structure (Figure 3A and B). HVMFs built a stable core spheroid surrounded by one to three layers of BeWo cells, which did not invade the fibroblastic core. Only in rare cases, BeWo cells did not cover the entire surface of the fibroblastic MTs. Interruptions of the syncytiotrophoblast barrier are also a regular finding, which occur in every placenta and throughout pregnancy ${ }^{21}$. It has been described that approximately $7 \%$ of the villous surface contains such gaps filled with fibrin as a result of blood clotting after degeneration or mechanical forces ${ }^{40}$. The established 3D co-culture MTs were highly viable as no necrotic core formation was evident on HE stained paraffin sections (Figure 3C). Furthermore, a live/dead staining confirmed the absence of dead cells (Figure S5). Upon staining for the proliferation marker MIB-1, co-culture MTs showed positive cells exclusively in the outer shell, indicating that BeWo cells continued to divide (Figure 3D). To 
see if a stable MT size can be obtained as previously observed for some cell lines ${ }^{24}$ we performed a growth profile of the co-culture MTs over 14 days starting on day 5 after MT formation was completed (Figure 4 and ESI Figure S3). While HVMF core MTs gradually compacted and obtained a stable size of $164 \pm 6.9 \mu \mathrm{m}$, BeWo/HVMF MTs steadily increased in size and exceeded a critical diameter of $500 \mu \mathrm{m}$ at day 10 . Since BeWo cells lack cell contact inhibition when cultivated in 2D, we explored if the use of HTR-8/SVneo trophoblast cells, which grow to a confluent monolayer could be used to obtain stable co-culture MTs. However, HTR-8/SVneo cells continued to proliferate in 3D co-culture MTs, albeit at a lower growth rate as compared to BeWo cells (Figure 4). In future studies, the replacement of BeWo cells by post-mitotic primary trophoblasts may provide a solution to obtain stable co-culture MTs for long-term exposure studies.

One of the major functions of the human placenta is the production of different hormones in order to support pregnancy and fetal growth. Among these, human chorionic gonadotropin (hCG) is a key hormone, which is produced by the syncytiotrophoblast of the chorionic villi and is secreted into the intervillous space. hCG fulfills many essential functions in pregnancy, including the promotion of progesterone production, implantation and decidualization, angiogenesis, cytotrophoblast differentiation, and immune cell regulation ${ }^{41}$. Potential hCG dysregulation could therefore lead to adverse pregnancy outcomes ${ }^{42}$. In this study, hCG levels were measured as an indicator for the functionality of BeWo cells and later on to study if NPs may interfere with endocrine functions of the placenta. When cultured in 3D co-culture MTs, BeWo cells released significantly more hCG as compared to 2D monocultures (Figure 5). Moreover, hCG production could be stimulated by forskolin, a known inducer of trophoblast differentiation. These results confirm that BeWo cells cultivated in 3D are highly functional. In addition, the significantly increased hCG levels suggest that BeWo cells may be in a more differentiated state towards the formation of a syncytium when grown under 3D culture conditions ${ }^{43}$. In order to visualize potential syncytium formation of BeWo cells on the MTs, tight junctions were immunofluorescently stained with an anti-ZO-1 antibody (ESI Figure S4). Small multinucleated areas with syncytia characteristics were observed in untreated control MTs and forskolin addition did not further increase the extent of syncytialization. However, the visualization of tight junctions in 3D MTs is challenging and further studies should be performed to unambiguously confirm syncytium formation on 3D MTs.

Single cell type spheroids of human choriocarcinoma cells have been established previously and are mostly used to study various aspects of implantation and placental physiology ${ }^{44,45}$. Introduction of a fibroblast core as in the here presented model was performed to better mimic the placental barrier structure, which is particularly relevant for NP uptake/penetration studies. Moreover, inclusion of fibroblasts is expected to provide more tissue-relevant responses since uptake and biological effects of NPs can be highly dependent on the cell type as well as cell type to cell type interactions ${ }^{46,47}$. Finally, hCG production in co-culture MTs is limited to the outer 
layers formed by BeWo cells similar to the syncytiotrophoblast layer covering the maternal surface of the placental tissue barrier. In mono-BeWo spheroids, cells in the core will be partially protected from NP exposure and may continue to produce hCG, thereby potentially masking subtle effects of NPs on hormone secretion.

\section{Nanoparticle selection and characterization}

Different NPs of industrial relevance were provided within the EU FP7 Nanosolutions project. We have selected non-toxic $\mathrm{TiO}_{2} \mathrm{NPs}^{48}$ as well as generally toxic $\mathrm{CuO}$ and $\mathrm{CdTe}-\mathrm{COOH} \mathrm{NPs}{ }^{49}, 50$ in order to evaluate the potential of the 3D MT model for nanotoxicity assessment. Different methods were applied to assess the physico-chemical characteristics of these NPs (for a summary on NP characteristics see Table S1 in the ESI). The average primary particle size determined by TEM was around $4 \mathrm{~nm}$ for $\mathrm{TiO}_{2}$ NPs and $10-20 \mathrm{~nm}$ for CuO NPs (ESI Figure S1). BET analysis revealed a specific surface area of $98 \pm 10 \mathrm{~m}^{2} / \mathrm{g}$ for $\mathrm{TiO}_{2} \mathrm{NPs}$ and of $42 \pm 2 \mathrm{~m}^{2} / \mathrm{g}$ for CuO NPs. The average particle size according to BET was $14 \mathrm{~nm}$ for $\mathrm{TiO}_{2}$ NPs and $20 \mathrm{~nm}$ for CuO NPs. Dynamic light scattering (DLS) analysis showed a hydrodynamic size of the dispersed particles of $17 \pm 0.3 \mathrm{~nm}$ for $\mathrm{TiO}_{2}$ and $1165 \pm 71 \mathrm{~nm}$ for CuO NPs in water and of $8284 \pm 900 \mathrm{~nm}$ for $\mathrm{TiO}_{2}$ and $1851 \pm 159$ $\mathrm{nm}$ for CuO NPs in medium, indicating a strong agglomeration of particles in medium. UV-Vis absorption and fluorescence spectroscopy was applied to characterize CdTe-COOH NP suspensions. Absorption spectra displayed a maximum located at $549 \mathrm{~nm}$ wavelength (ESI Figure S2), while the fluorescence emission spectra were characterized by a peak at $580 \mathrm{~nm}$ wavelength (data not shown). The diameter of $\mathrm{CdTe}-\mathrm{COOH}$ NPs as calculated from the position of the exciton band was around $3.2 \mathrm{~nm}{ }^{42}$. These values are typical for CdTe NPs, and together with the high fluorescence quantum yield, indicate that the $\mathrm{CdTe}-\mathrm{COOH}$ NPs were well dispersed in the aqueous suspension. All NPs have been tested for endotoxin contamination and were endotoxin free ( $<0.5 \mathrm{EU} / \mathrm{ml}$ in Chromogenic LAL assay).

\section{Impact of the selected NPs on placental co-culture MT viability}

The effects of $\mathrm{TiO}_{2}, \mathrm{CuO}$ or $\mathrm{CdTe}-\mathrm{COOH}$ NPs on co-culture MT viability were quantified using a luminescent cell viability assay, which measures the intracellular ATP content. In this assay, MTs are lysed before ATP is determined, which results in a higher sensitivity as compared to e.g. the MTS assay where the diffusion of the substrate is limited by the 3D structure. While $\mathrm{TiO}_{2} \mathrm{NPs}$ did not affect MT viability, $\mathrm{CdTe}-\mathrm{COOH}$ and $\mathrm{CuO}$ NPs induced a dose-dependent decrease in ATP levels (Figure 6). $\mathrm{CdTe}-\mathrm{COOH}$ NPs were slightly more toxic to co-culture MTs than CuO NPs. The cytotoxic effects of $\mathrm{CuO}$ and $\mathrm{CdTe}-\mathrm{COOH}$ NPs were also observed when performing the same ATP assay in 2D HVMF or BeWo monocultures (ESI Figure S5). The reduction in ATP levels was highly similar for both cell types, suggesting that HVMF and BeWo cells are equally sensitive to 
$\mathrm{CuO}$ and $\mathrm{CdTe}-\mathrm{NPs}$. However, $\mathrm{EC}_{50}$ values for $\mathrm{CuO}$ and $\mathrm{CdTe}-\mathrm{COOH}$ NPs were significantly higher for 3D MTs $(9.34 \mu \mathrm{g} / \mathrm{ml}$ and $27.3 \mu \mathrm{g} / \mathrm{ml})$ than for 2D monocultures $(6.41 \mu \mathrm{g} / \mathrm{ml}$ and $8.62 \mu \mathrm{g} / \mathrm{ml}$ for HVMFs or $2.9 \mu \mathrm{g} / \mathrm{ml}$ and $6.51 \mu \mathrm{g} / \mathrm{ml}$ for BeWo cells). These results suggest that 3D placental MTs are more resistant to toxic NPs than 2D cell cultures. Yet, a direct comparison is challenging due to differences in cell numbers or the use of mono- versus co-cultures, among others. Nevertheless, an increased resistance of 3D MTs to toxic NPs has been previously observed such as for liver MTs exposed to CdTe NPs ${ }^{14}$, colon MTs exposed to Zn NPs ${ }^{51}$ or MTs from human adipose-derived mesenchymal stem cells exposed to carboxyl-functionalized $\mathrm{CdTe} / \mathrm{CdS} / \mathrm{ZnS}$ Qdots ${ }^{52}$. The differences are likely due to the fact that close cell-cell and cell-matrix interactions as well as the tissue-like structure provided by 3D MTs limit the penetration of particles. Consequently, cells in the core of the MTs will be partially protected from the exposure to toxic NPs by the cells of the outer layers ${ }^{24,52}$. In the case of the placental co-culture MTs, BeWo cells will be preferentially affected by the NPs due to the core-shell structure with BeWo cells on the outer edge of the spheroid. Indeed, live/dead staining confirmed that mostly the outer cells were dying after exposure to increasing concentrations of CuO NPs whereas cells in the core remained viable (ESI Figure S6). Therefore, even if the viability of 3D MTs is only slightly reduced by a few percentages, placental barrier integrity may already be severely compromised. As CuO and CdTe$\mathrm{COOH}$ induced major cytotoxicity on HVMF and BeWo cells both in 2D monocultures as well as in 3D co-cultures the exposure to such NPs during pregnancy should be avoided. Adverse effects were also observed in many other in vitro and in vivo studies after exposure to CuO NPs ${ }^{53-56}$ or semiconducting NPs (e.g. CdTe ${ }^{57-59}$ or CdSe/ZnS 60, 61).

It appears that the mechanisms underlying the toxicity of these NPs include particle dissolution and/or the generation of reactive oxygen species (ROS) $55,56,58,60,62,63$. Leaching of toxic ions from NPs can occur outside of cells or intracellularly, in particular in lysosomes where a low pH favors dissolution (Trojan horse mechanism) ${ }^{64}$. Free cadmium and copper ions act as catalysts in the formation of ROS, which may result in oxidative tissue damage ${ }^{65,66}$. Dissolution studies of $\mathrm{TiO}_{2}$ and CuO NPs were performed in cell culture medium and ions were separated from NPs by centrifugation. $\mathrm{CdTe}-\mathrm{COOH}$ NPs were not included as they were too small to remove particles by centrifugation or ultrafiltration. We measured only a minor release of $\mathrm{Ti}$ ions from $\mathrm{TiO}_{2} \mathrm{NPs}$ $(1.5 \%$ Ti after $24 \mathrm{~h}$ ) whereas the percentage of $\mathrm{Cu}$ ions was $13 \%$ after $10 \mathrm{~min}$ and $59 \%$ after 24 $h$ of incubation (ESI Table S1). Since centrifugation might not remove all NPs from the supernatant, ultrafiltration was used as an alternative method. The amount of released $\mathrm{Cu}$ ions was $7 \%$ after $10 \mathrm{~min}$ and $50 \%$ after $24 \mathrm{~h}$ of incubation, which was highly similar to the results obtained by centrifugation. Although solubility studies in the absence of cells do not fully reflect the experimental conditions, the high dissolution of CuO NPs in medium suggests that toxic $\mathrm{Cu}$ ions are at least partially involved in CuO NP-mediated toxicity to placental cells. To understand if the 
absence of cytotoxic effects upon exposure to $\mathrm{TiO}_{2} \mathrm{NPs}$ was due to the lack of particle uptake we performed TEM analysis. $\mathrm{TiO}_{2}$ NPs were internalized by BeWo cells and large agglomerates were present either freely in the cytoplasm or in membrane-bound vesicles (ESI Figure S7). However, no $\mathrm{TiO}_{2} \mathrm{NPs}$ were observed in the fibroblastic core. We do not exclude that single $\mathrm{TiO}_{2} \mathrm{NPs}$ or small agglomerates may have penetrated into the fibroblastic core but as this area is highly compact and electron dense (ESI Figure S8), it is much more difficult to detect internalized particles in the MT core.

Validation of the obtained results in order to understand the predictive value of the co-culture MTs is challenging as only few in vivo data are available from animal studies, which may not be representative for human pregnancy due to major species-specific differences. In mice, 1-3 nm $\mathrm{CdTe} / \mathrm{CdS}$ Qdots and $35 \mathrm{~nm} \mathrm{TiO} 2$ NPs appeared to cross the placental barrier, although in very low amounts ${ }^{8,67}$. While no histological defects to the placental tissue were observed after Qdot exposure ${ }^{8}, \mathrm{TiO}_{2}$ NPs affected tissue viability and structure ${ }^{67}$. However, to circumvent uncertainties due to the use of different NPs or rodent models, we performed additional ex vivo human placenta perfusion studies with $\mathrm{TiO}_{2}$ NPs used for co-culture MTs exposure (ESI Figure S9). Ti concentrations determined in the fetal circuit were only $1 \%$ of the initial dose applied to the maternal circuit. However, similar Ti concentrations were also measured in control perfusions where no $\mathrm{TiO}_{2} \mathrm{NPs}$ were applied (data not shown), indicating that $\mathrm{TiO}_{2} \mathrm{NP}$ translocation was very low or even absent. The measured Ti background signal of approximately $0.2 \mu \mathrm{g} / \mathrm{g}$ was still above the detection limit $(0.001 \mu \mathrm{g} / \mathrm{g})$. In the maternal circulation, Ti levels decreased to $20 \%$ of the initial amount suggesting that particles agglomerated in the placental tissue. The absence of vascular leakage and NP transfer indicates that $\mathrm{TiO}_{2} \mathrm{NPs}$ did not elicit major damage to placental barrier integrity. Similar conclusions have been obtained from our co-culture MT studies where $\mathrm{TiO}_{2}$ NPs did accumulate in the trophoblast layer (ESI Figure S7) but did not affect BeWo cell viability (Figure 6). From these comparisons it appears that co-culture placenta MTs may indeed be a valuable model to assess NP-placenta interactions, which could then be further validated in ex vivo perfusion or animal studies. However, to fully understand the predictive value and limitations of placental co-culture MTs further characterization and validation studies will be required.

\section{Impact of the selected NPs on the secretion of hCG from placental co-culture MTs}

Even if NPs do not elicit direct cytotoxic effects on the placental tissue they might affect fetal health if they interfere with proper placental function. A hugely important function of the human placenta is the production of many hormones, which affect the status of pregnancy and maternal 
physiology. A malfunction may result in fetal complications or even a miscarriage ${ }^{42}$. To understand if $\mathrm{TiO}_{2}, \mathrm{CdTe}-\mathrm{COOH}$ or $\mathrm{CuO}$ NPs may act as endocrine disruptors at the placental barrier, we assessed their effects on the release of hCG in 3D co-culture MTs. These studies were performed in the presence of $20 \mu \mathrm{M}$ forskolin in order to upregulate the differentiation of BeWo cells and to allow a decreases in hCG levels to be easily detected. $\mathrm{TiO}_{2}$ NPs did not affect hCG levels whereas already low concentrations of $\mathrm{CuO}$ or $\mathrm{CdTe-COOH} \mathrm{NPs} \mathrm{significantly} \mathrm{reduced} \mathrm{hCG}$ production (Figure 7). However, it was difficult to identify subtoxic NP doses, which was required to exclude that the observed reduction was a secondary effect due to cell death. Since hCG in co-culture MTs is only produced by BeWo cells, dose-response curves for the individual cell types in the co-culture MTs would be required. Attempts to obtain such toxicity data using FACS anal-

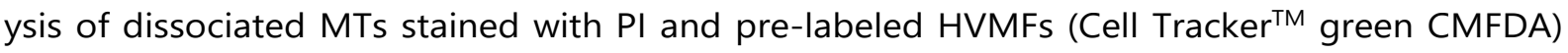
failed due to non-specific staining of BeWo cells with dye released from dead HVMFs. Nevertheless, live/dead staining and microscopic images of the co-culture MTs after exposure to CuO NPs showed that $0.5 \mu \mathrm{g} / \mathrm{ml}$ was likely to reflect a non-toxic concentration since only few dead cells were present on

the MT surface and the edge of the MTs was still compact (ESI Figure S6A and B). No live/dead images were possible for $\mathrm{CdTe}-\mathrm{COOH}$ NPs due to their fluorescent properties but bright-field images revealed MTs with a compact surface for concentrations up to $3 \mu \mathrm{g} / \mathrm{ml}$ (ESI Figure S6C). In addition, ATP assay of 2D BeWo monocultures showed that after treatment with $0.5 \mu \mathrm{g} / \mathrm{ml}$ $\mathrm{CuO} \mathrm{NPs}$ or $3 \mu \mathrm{g} / \mathrm{ml} \mathrm{CdTe-COOH} \mathrm{NPs,} \mathrm{cell} \mathrm{viability} \mathrm{was} \mathrm{around} 80 \%$ or higher, indicating that these are likely to reflect subtoxic concentrations.

At $3 \mu \mathrm{g} / \mathrm{ml} \mathrm{CdTe-COOH} \mathrm{NPs,} \mathrm{hCG} \mathrm{levels} \mathrm{were} \mathrm{significantly} \mathrm{decreased} \mathrm{whereas} \mathrm{only} \mathrm{a} \mathrm{slight} \mathrm{(non-}$ significant) reduction was observed for $0.5 \mu \mathrm{g} / \mathrm{ml}$ CuO NPs (Figure 7). Collectively, these findings suggest that $\mathrm{CdTe}-\mathrm{COOH}$ NPs may have the potential to interfere with the endocrine function of the placenta but further studies including additional placenta-relevant hormones as well as in vivo studies would be required to corroborate this hypothesis. Interestingly, there is supporting evidence from studies on smoking women showing that exposure to $\mathrm{Cd}$ and other toxic metals interferes with placental hormone production (e.g. Cd affects placental leptin and progesterone production ${ }^{68}$ ).

\section{Conclusions}

During the last years, a large variety of highly sophisticated 3D models have been developed for different biological tissues and barrier such as the lung ${ }^{69-71}$, the liver ${ }^{72}$ or the brain ${ }^{73}$. These models achieved major improvements in regard to toxicity assessment of drugs or NPs as compared to 2D models. However, 3D models of the placental barrier are scarce despite the urgent need for reliable nanosafety data in human pregnancy $1,2,74$. With the development of the first 
human 3D placental co-culture microtissue model using the high-throughput compatible hanging drop technology we enable a more realistic mechanistic toxicity assessment at tissue level. Cell morphology, hormone secretion by trophoblasts and structural organization of the co-culture MTs were shown to better recapitulate the in vivo situation as compared to 2D cell cultures. We confirmed that this model is suitable for the rapid evaluation of acute toxicity and hormone secretion levels after the exposure to different NPs. Importantly, our results on $\mathrm{CdTe}-\mathrm{COOH}$ NPs showed that for core/shell-type co-culture MTs it is critical to not only study cell type-independent endpoints such as ATP levels as these may underestimate NP toxicity at the placental barrier. To obtain a fundamental understanding of NP-placenta interactions, it will be critical to relate adverse effects to the individual cell types and/or to include cell type-specific measurements such as hormone production. In addition to acute toxicity assessment, the co-culture MT model holds great potential for the assessment of NP penetration and uptake mechanisms because a more tissue-like transport of NPs can be expected due to the 3D structure. Finally, we anticipate that the inclusion of additional cell types such as immune cells or endothelial cells as well as the replacement of BeWo cells with primary trophoblasts will further improve the predictive value of this promising new 3D placental microtissue model.

\section{Acknowledgements}

We thank Dr. Matthias Roesslein, Empa, for support in statistical analysis and Prof. Bengt Fadeel, Karolinska Institutet, for helpful comments on the manuscript. This project has received funding from the European Union's Seventh Framework Programme for research, technological development and demonstration under grant agreement no 309329 (NANOSOLUTIONS) and no 263215 (MARINA).

\section{References}

1 C. Muoth, L. Aengenheister, M. Kucki, P. Wick and T. Buerki-Thurnherr, Nanomedicine, 2016, 11, 941-957.

2 J. A. Keelan, J. W. Leong, D. Ho and K. S. Iyer, Nanomedicine, 2015, 10, 2229-2247.

3 W. Qi, J. Bi, X. Zhang, J. Wang, J. Wang, P. Liu, Z. Li and W. Wu, Sci Rep, 2014, 4, 4352.

4 X. L. Huang, F. Zhang, X. L. Sun, K. Y. Choi, G. Niu, G. F. Zhang, J. X. Guo, S. Lee and X. Y. Chen, Biomaterials, 2014, 35, 856-865.

5 L. Campagnolo, M. Massimiani, G. Palmieri, R. Bernardini, C. Sacchetti, A. Bergamaschi, L. Vecchione, A. Magrini, M. Bottini and A. Pietroiusti, Part Fibre Toxicol, 2013, 10, 21.

6 A. Pietroiusti, M. Massimiani, I. Fenoglio, M. Colonna, F. Valentini, G. Palleschi, A. Camaioni, A. Magrini, G. Siracusa, A. Bergamaschi, A. Sgambato and L. Campagnolo, Acs Nano, 2011, 5, 4624-4633.

7 J. L. Blum, J. Q. Xiong, C. Hoffman and J. T. Zelikoff, Toxicol Sci, 2012, 126, 478-486.

8 M. Chu, Q. Wu, H. Yang, R. Yuan, S. Hou, Y. Yang, Y. Zou, S. Xu, K. Xu, A. Ji and L. Sheng, Small, 2010, 6, 670678.

9 A. Adamcakova-Dodd, M. M. Monick, L. S. Powers, K. N. Gibson-Corley and P. S. Thorne, Part Fibre Toxicol, 2015, 12, 30.

10 T. Buerki-Thurnherr, U. von Mandach and P. Wick, Swiss medical weekly, 2012, 142, w13559.

11 A. Malassine, J. L. Frendo and D. Evain-Brion, Hum Reprod Update, 2003, 9, 531-539.

12 S. Grafmueller, P. Manser, H. F. Krug, P. Wick and U. von Mandach, J. Vis. Exp., 2013, DOI: doi:10.3791/50401, e50401.

13 J. R. Hutson, F. Garcia-Bournissen, A. Davis and G. Koren, Clin Pharmacol Ther, 2011, 90, 67-76.

14 J. Lee, G. D. Lilly, R. C. Doty, P. Podsiadlo and N. A. Kotov, Small, 2009, 5, 1213-1221. 
15 K. M. Yamada and E. Cukierman, Cell, 2007, 130, 601-610.

16 E. R. Shamir and A. J. Ewald, Nat Rev Mol Cell Biol, 2014, 15, 647-664.

17 K. S. Smalley, M. Lioni and M. Herlyn, In Vitro Cell Dev Biol Anim, 2006, 42, 242-247.

18 F. Pampaloni, E. G. Reynaud and E. H. Stelzer, Nat Rev Mol Cell Biol, 2007, 8, 839-845.

19 A. I. Minchinton and I. F. Tannock, Nat Rev Cancer, 2006, 6, 583-592.

20 F. Hirschhaeuser, H. Menne, C. Dittfeld, J. West, W. Mueller-Klieser and L. A. Kunz-Schughart, J Biotechnol, 2010, 148, 3-15.

21 P. Kaufmann, B. Huppertz and H.-G. Frank, Annals of Anatomy - Anatomischer Anzeiger, 1996, 178, 485501.

22 D. T. Leong and K. W. Ng, Adv Drug Deliv Rev, 2014, 79-80, 95-106.

23 A. Kermanizadeh, M. Lohr, M. Roursgaard, S. Messner, P. Gunness, J. M. Kelm, P. Moller, V. Stone and S. Loft, Part Fibre Toxicol, 2014, 11, 56.

24 Y. Luo, C. Wang, M. Hossain, Y. Qiao, L. Ma, J. An and M. Su, Anal Chem, 2012, 84, 6731-6738.

25 25. T. T. Goodman, P. L. Olive and S. H. Pun, Int J Nanomedicine, 2007, 2, 265-274.

26 K. Huang, H. Ma, J. Liu, S. Huo, A. Kumar, T. Wei, X. Zhang, S. Jin, Y. Gan, P. C. Wang, S. He, X. Zhang and X. J. Liang, ACS Nano, 2012, 6, 4483-4493.

27 A. S. Mikhail, S. Eetezadi, S. N. Ekdawi, J. Stewart and C. Allen, Int J Pharm, 2014, 464, 168-177.

28 J. M. Kelm and M. Fussenegger, Trends in Biotechnology, 2004, 22, 195-202.

29 C. H. Graham, T. S. Hawley, R. G. Hawley, J. R. MacDougall, R. S. Kerbel, N. Khoo and P. K. Lala, Exp Cell Res, 1993, 206, 204-211.

30 J. M. Kelm, N. E. Timmins, C. J. Brown, M. Fussenegger and L. K. Nielsen, Biotechnology and bioengineering, $2003,83,173-180$.

31 J. S. Taurozzi, V. A. Hackley and M. R. Wiesner, Nanotoxicology, 2011, 5, 711-729.

32 A. Malek, R. Sager, A. B. Lang and H. Schneider, Am J Reprod Immunol, 1997, 38, 263-271.

33 M. Rimann and U. Graf-Hausner, Curr Opin Biotechnol, 2012, 23, 803-809.

34 J. M. Kelm and M. Fussenegger, Trends Biotechnol, 2004, 22, 195-202.

35 P. Kaufmann, J. Stark and H. E. Stegner, Cell Tissue Res, 1977, 177, 105-121.

36 M. Castellucci and P. Kaufmann, in Pathology of the Human Placenta, 5 edn., 2006, pp. 50 - 120.

37 D. A. Evseenko, J. W. Paxton and J. A. Keelan, American journal of physiology. Regulatory, integrative and comparative physiology, 2006, 290, R1357-1365.

38 K. Orendi, V. Kivity, M. Sammar, Y. Grimpel, R. Gonen, H. Meiri, E. Lubzens and B. Huppertz, Placenta, 2011, 32 Suppl, S49-54.

39 K. Orendi, M. Gauster, G. Moser, H. Meiri and B. Huppertz, Reproduction, 2010, 140, 759-766.

40 D. M. Nelson, Placenta, 1996, 17, 387-391.

41 L. A. Cole, Reprod Biol Endocrinol, 2010, 8, 102.

42 W. Norris, T. Nevers, S. Sharma and S. Kalkunte, Placenta, 2011, 32 Suppl 2, S182-185.

43 T. M. Butler, P. A. Elustondo, G. E. Hannigan and D. J. MacPhee, Reprod Biol Endocrinol, 2009, 7, 51.

44 R. Grümmer, H. P. Hohn, M. M. Mareel and H. W. Denker, Placenta, 1994, 15, 411-429.

45 R. Grümmer, H.-P. Hohn and H.-W. Denker, in Trophoblast Invasion and Endometrial Receptivity: Novel Aspects of the Cell Biology of Embryo Implantation, eds. H.-W. Denker and J. D. Aplin, Springer US, Boston, MA, 1990, DOI: 10.1007/978-1-4613-0615-3_5, pp. 97-111.

46 I. Y. Kim, E. Joachim, H. Choi and K. Kim, Nanomedicine, 2015, 11, 1407-1416

47 M. J. Clift, C. Endes, D. Vanhecke, P. Wick, P. Gehr, R. P. Schins, A. Petri-Fink and B. Rothen-Rutishauser, Toxicol Sci, 2014, 137, 55-64.

48 L. Farcal, F. Torres Andon, L. Di Cristo, B. M. Rotoli, O. Bussolati, E. Bergamaschi, A. Mech, N. B. Hartmann, K. Rasmussen, J. Riego-Sintes, J. Ponti, A. Kinsner-Ovaskainen, F. Rossi, A. Oomen, P. Bos, R. Chen, R. Bai, C. Chen, L. Rocks, N. Fulton, B. Ross, G. Hutchison, L. Tran, S. Mues, R. Ossig, J. Schnekenburger, L. Campagnolo, L. Vecchione, A. Pietroiusti and B. Fadeel, PLoS One, 2015, 10, e0127174.

49 R. Hardman, Environ Health Perspect, 2006, 114, 165-172.

50 A. Ivask, K. Juganson, O. Bondarenko, M. Mortimer, V. Aruoja, K. Kasemets, I. Blinova, M. Heinlaan, V. Slaveykova and A. Kahru, Nanotoxicology, 2014, 8 Suppl 1, 57-71.

51 S. L. Chia, C. Y. Tay, M. I. Setyawati and D. T. Leong, Small, 2015, 11, 702-712.

52 M. Ulusoy, A. Lavrentieva, J.-G. Walter, F. Sambale, M. Green, F. Stahl and T. Scheper, Toxicol. Res., 2016, 5, 126-135.

53 M. Ahamed, M. J. Akhtar, H. A. Alhadlaq and S. A. Alrokayan, Nanomedicine (Lond), 2015, 10, $2365-2377$.

54 M. Caicedo, J. J. Jacobs, A. Reddy and N. J. Hallab, J Biomed Mater Res A, 2008, 86, 905-913.

55 B. Fahmy and S. A. Cormier, Toxicol In Vitro, 2009, 23, 1365-1371.

56 K. Midander, P. Cronholm, H. L. Karlsson, K. Elihn, L. Moller, C. Leygraf and I. O. Wallinder, Small, 2009, 5, 389-399.

57 57. X. Li, X. Yang, L. Yuwen, W. Yang, L. Weng, Z. Teng and L. Wang, Biomaterials, 2016, 96, 24-32.

58 J. Lovric, S. J. Cho, F. M. Winnik and D. Maysinger, Chem Biol, 2005, 12, 1227-1234.

59 T. Zhang, Y. Hu, M. Tang, L. Kong, J. Ying, T. Wu, Y. Xue and Y. Pu, Int J Mol Sci, 2015, 16, 23279-23299.

60 Y. Lu, S. Xu, H. Chen, M. He, Y. Deng, Z. Cao, H. Pi, C. Chen, M. Li, Q. Ma, P. Gao, Y. Ji, L. Zhang, Z. Yu and Z. Zhou, Biomaterials, 2016, 90, 27-39.

61 X. Wang, J. Tian, K. T. Yong, X. Zhu, M. C. Lin, W. Jiang, J. Li, Q. Huang and G. Lin, J Nanobiotechnology, 2016, 14, 10.

62 A. M. Derfus, W. C. W. Chan and S. N. Bhatia, Nano Letters, 2004, 4, 11-18.

63 C. Kirchner, A. M. Javier, A. S. Susha, A. L. Rogach, O. Kreft, G. B. Sukhorukov and W. J. Parak, Talanta, 2005, 67, 486-491.

64 L. K. Limbach, Y. Li, R. N. Grass, T. J. Brunner, M. A. Hintermann, M. Muller, D. Gunther and W. J. Stark, Environmental science \& technology, 2005, 39, 9370-9376. 
65 E. Birben, U. M. Sahiner, C. Sackesen, S. Erzurum and O. Kalayci, The World Allergy Organization journal, 2012, 5, 9-19.

66 S. J. Stohs and D. Bagchi, Free radical biology \& medicine, 1995, 18, 321-336.

67 K. Yamashita, Y. Yoshioka, K. Higashisaka, K. Mimura, Y. Morishita, M. Nozaki, T. Yoshida, T. Ogura, H. Nabeshi, K. Nagano, Y. Abe, H. Kamada, Y. Monobe, T. Imazawa, H. Aoshima, K. Shishido, Y. Kawai, T. Mayumi, S. Tsunoda, N. Itoh, T. Yoshikawa, I. Yanagihara, S. Saito and Y. Tsutsumi, Nat Nanotechnol, 2011, 6, 321328.

68 S. Stasenko, E. M. Bradford, M. Piasek, M. C. Henson, V. M. Varnai, J. Jurasović and V. Kušec, Journal of Applied Toxicology, 2010, 30, 242-253.

69 C. Jud, S. Ahmed, L. Müller, C. Kinnear, D. Vanhecke, Y. Umehara, S. Frey, M. Liley, S. Angeloni, A. Petri-Fink and B. Rothen-Rutishauser, BioResearch Open Access, 2015, 4, 457-468.

70 D. Huh, B. D. Matthews, A. Mammoto, M. Montoya-Zavala, H. Y. Hsin and D. E. Ingber, Science, 2010, 328, $1662-1668$

71 S. G. Klein, T. Serchi, L. Hoffmann, B. Blomeke and A. C. Gutleb, Part Fibre Toxicol, 2013, 10, 31.

72 S. Messner, I. Agarkova, W. Moritz and J. M. Kelm, Archives of toxicology, 2013, 87, 209-213.

73 E. Urich, C. Patsch, S. Aigner, M. Graf, R. lacone and P. O. Freskgard, Sci Rep, 2013, 3, 1500.

74 K. S. Hougaard, L. Campagnolo, P. Chavatte-Palmer, A. Tarrade, D. Rousseau-Ralliard, S. Valentino, M. V. Park, W. H. de Jong, G. Wolterink, A. H. Piersma, B. L. Ross, G. R. Hutchison, J. S. Hansen, U. Vogel, P. Jackson, R. Slama, A. Pietroiusti and F. R. Cassee, Reprod Toxicol, 2015, 56, 118-140.

75 S. Grafmueller, P. Manser, L. Diener, P. A. Diener, X. Maeder-Althaus, L. Maurizi, W. Jochum, H. F. Krug, T. Buerki-Thurnherr, U. von Mandach and P. Wick, Environ Health Perspect, 2015, 2015, 123, 1280-1286.

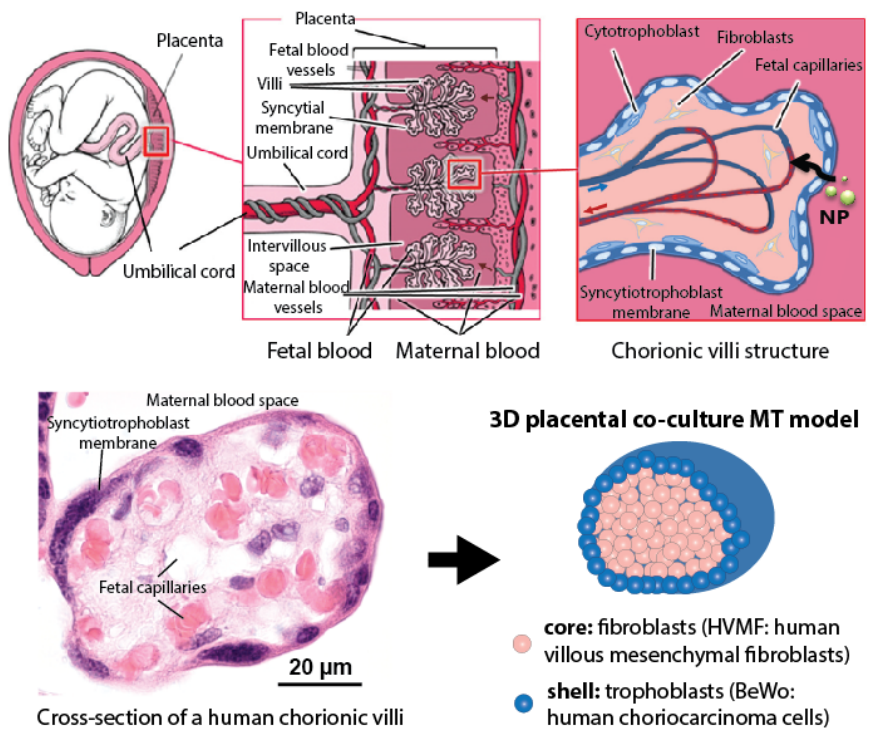

Figure 1. Scheme of the human placenta and MT model. In the intervillous space, chorionic villi are surrounded by maternal blood that provides gases and nutrients for the fetus. In order to enter the fetal blood stream, NPs first have to cross the syncytiotrophoblast layer that confine the villi, penetrate through the villous stroma containing fibroblasts and finally cross the fetal capillary endothelium. Our newly developed 3D co-culture MT model is composed of an inner core of fibroblasts that derive from the villous stroma (rose) surrounded by trophoblasts (blue), which contribute most to the specific barrier function of the placenta. Figure 1. was reproduced and adapted with permission from Nanomedicine as agreed by Future Medicine Ltd. ${ }^{2}$ 

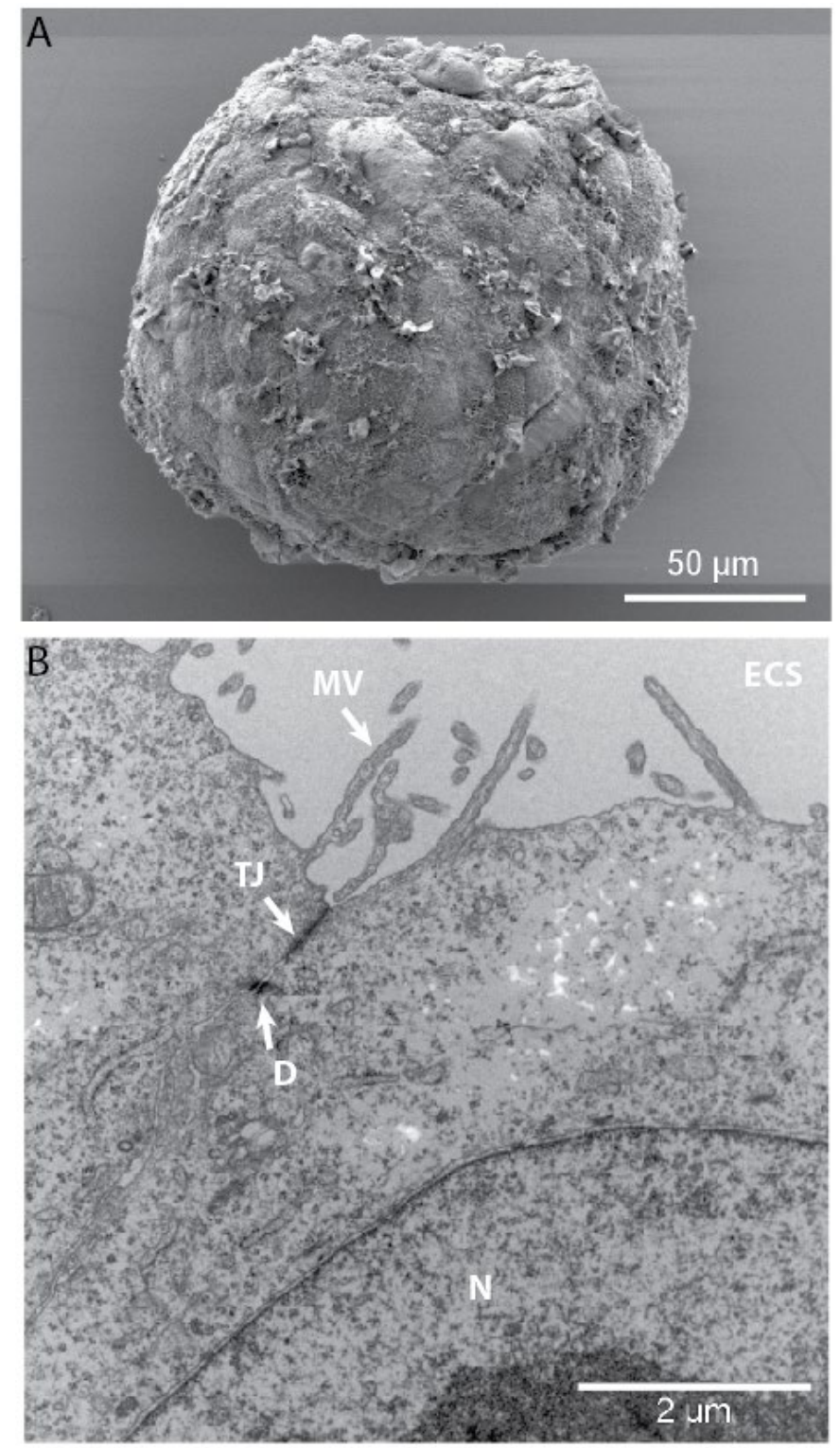

Figure 2. Electron microscopy of human placental co-culture MTs. After 5 days of cultivation, MTs were processed for SEM or TEM analysis. (A) Representative SEM image of a 3D co-culture MT. (B) TEM micrograph of BeWo cells on the edge of a 3D co-culture MT. Abbreviations: ECS, extra cellular space; $\mathrm{D}$, desmosome; N, nucleus; MV, microvilli; TJ, tight junction. 

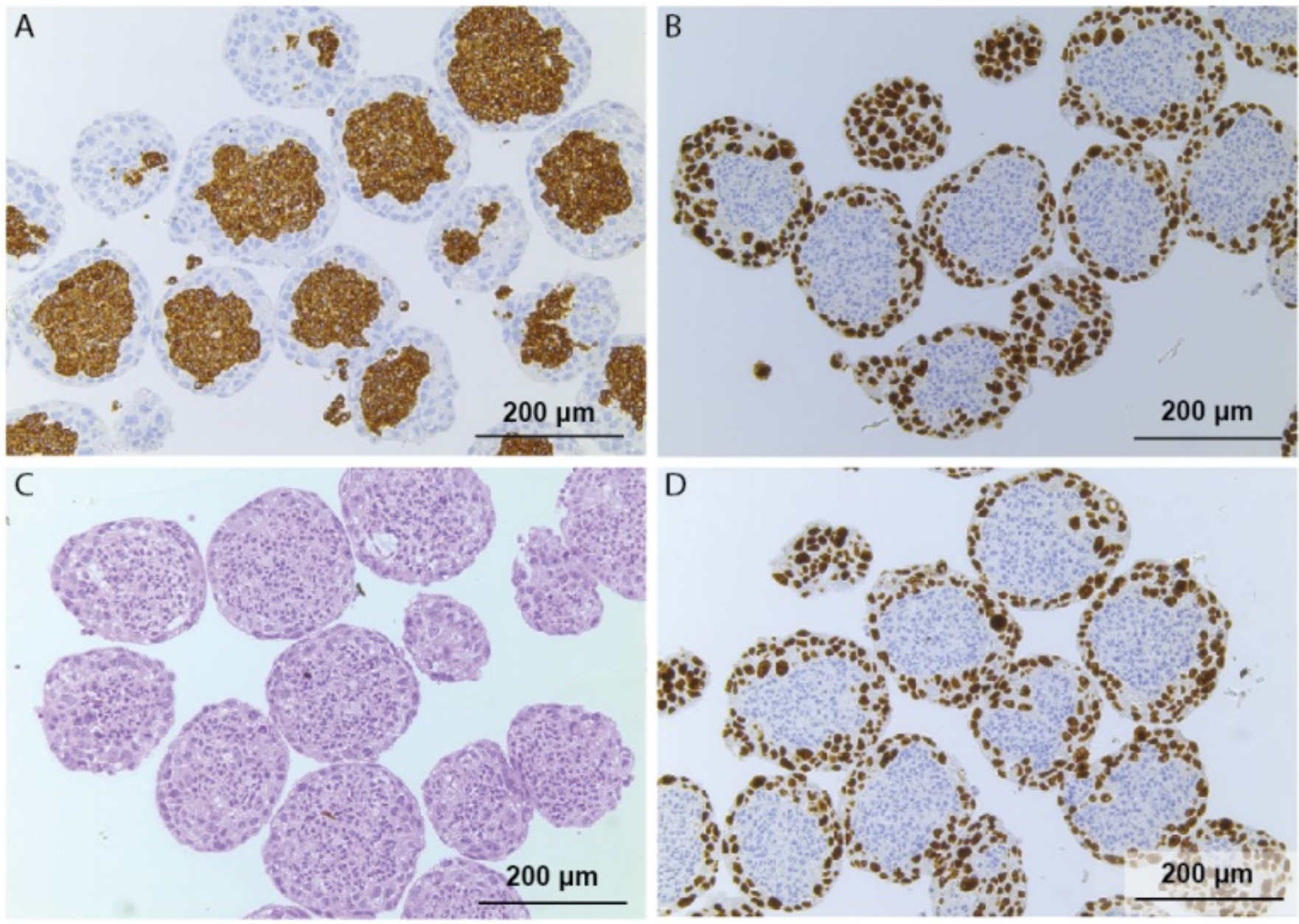

Figuree 3. Immunohistochemical characterization of human placental co-culture MTs. After 5 days of cultivation, MTs were processed for immunohistochemical analysis. Paraffin sections were stained with either the mesenchymal cell marker vimentin to visualize HVMF (A), the epithelial cell marker MNF116 to label cytokeratins specifically expressed by BeWo cells (B), hematoxylin/eosin (C) or MIB-1 to show proliferating cells (D).

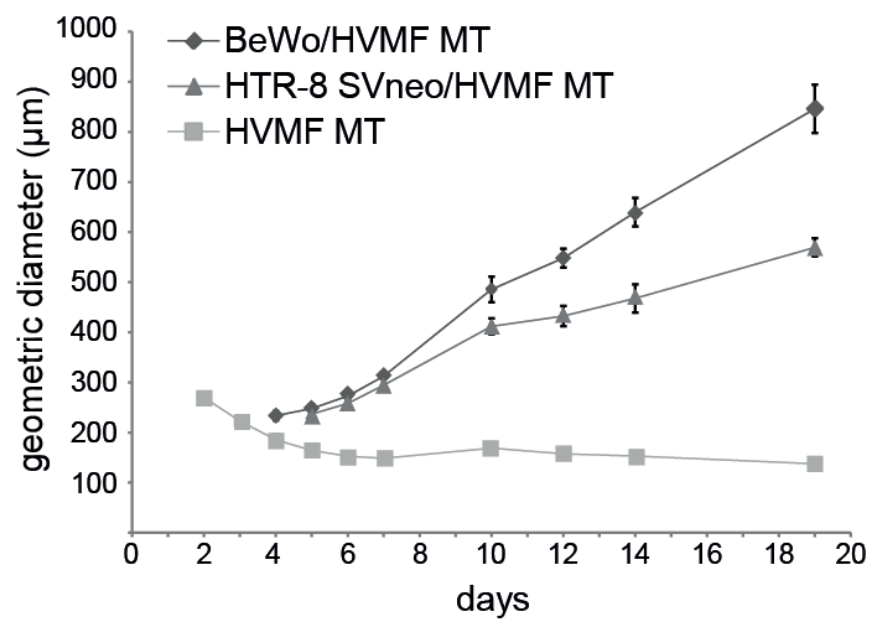

Figure 4. Growth profiles of fibroblastic core MTs and co-culture MTs over 19 days. The geometric diameter was calculated for HVMF, BeWo/HVMF and HTR-8/SVneo/HVMF co-culture MTs. 


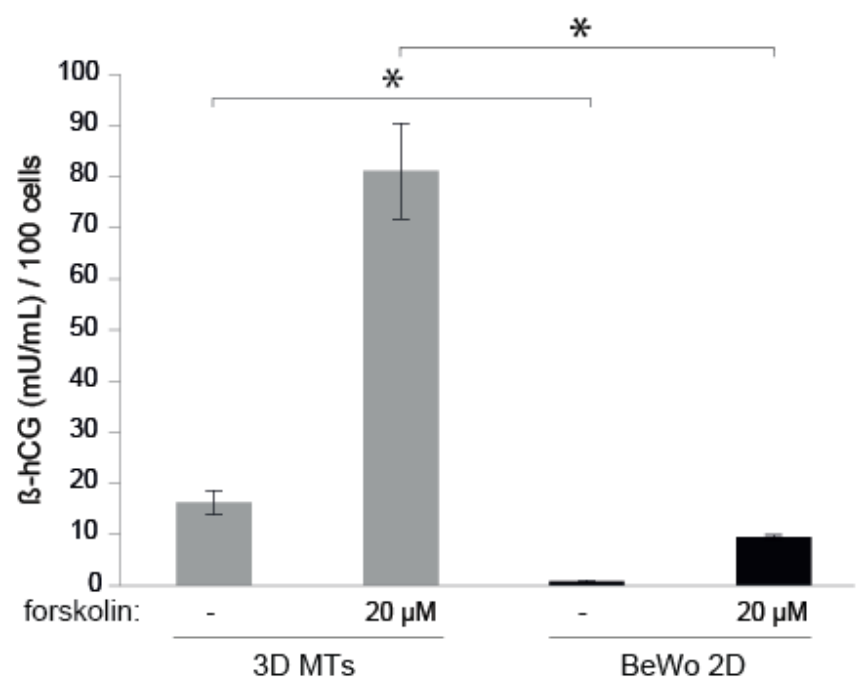

Figure 5. Functional characterization of 3D MTs for the production of B-hCG and comparison to 2D BeWo monocultures. The release of the placental hormone B-hCG from BeWo cells grown in 3D co-culture MTs or as 2D monocultures was determined after $24 \mathrm{~h}$ of cultivation with and without stimulation of BeWo cell differentiation with $20 \mu \mathrm{M}$ forskolin.

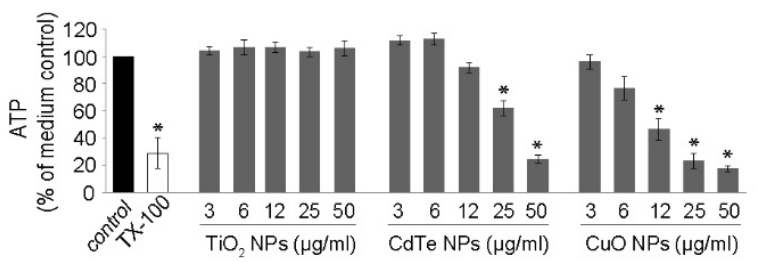

Figure 6. Effects of different NPs on the 3D MT viability. Co-culture MTs were exposed to $\mathrm{TiO}_{2}, \mathrm{CdTe}-\mathrm{COOH}$ or CuO NPs for $24 \mathrm{~h}$. Cell viability was assessed using the ATP assay. As a positive control cells were treated with $0.1 \%$ TX-100. Data represent the mean percentages triplicates compared to the untreated medium control \pm SEM of 3 4 independent experiments. ${ }^{*} p<0.05$ compared to untreated medium control

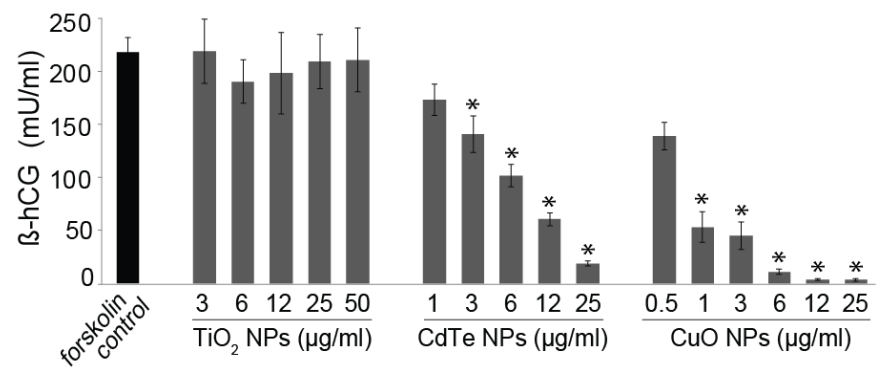

Figure 7. B-hCG levels of co-culture MTs after exposure to different NPs. MTs were exposed to TiO2, CdTe$\mathrm{COOH}$ or CuO NPs diluted in medium containing $20 \mu \mathrm{M}$ forskolin for $24 \mathrm{~h}$. Data represent the mean \pm SEM (NPs $\mathrm{n}$ $=3-4$, forskolin control $n=14)$. ${ }^{*} p<0.05$ vs $20 \mu \mathrm{M}$ forskolin control 